\title{
COMPACT NON-ORIENTABLE SURFACES OF GENUS 6 WITH EXTREMAL METRIC DISCS
}

\begin{abstract}
GOU NAKAMURA
Dedicated to Professor Noriaki Suzuki on the occasion of his sixtieth birthday

ABSTRACT. A compact hyperbolic surface of genus $g$ is said to be extremal if it admits an extremal disc, a disc of the largest radius determined only by $g$. We discuss how many extremal discs are embedded in non-orientable extremal surfaces of genus 6 . This is the final genus in our interest because it is already known for $g=3,4,5$, or $g>6$. We show that non-orientable extremal surfaces of genus 6 admit at most two extremal discs. The locus of extremal discs is also obtained for each surface. Consequently non-orientable extremal surfaces of arbitrary genus $g \geqq 3$ admit at most two extremal discs. Furthermore we determine the groups of automorphisms of non-orientable extremal surfaces of genus 6 with two extremal discs.
\end{abstract}

\section{INTRODUCTION}

Let $S$ be a compact hyperbolic surface $S$ of genus $g$, where $g$ denotes the number of handles $(g \geqq 2)$ if $S$ is orientable, or the number of cross caps $(g \geqq 3)$ if $S$ is non-orientable. The hyperbolic metric on $S$ is the one induced by the hyperbolic metric defined by the differential $d s=2|d z| /\left(1-|z|^{2}\right)$ on the unit disc $\mathbb{D}$. In [2] C. Bavard showed that the radius $r$ of a disc embedded in $S$ satisfies the inequality

$$
\cosh r \leqq \frac{1}{2 \sin \frac{\pi}{6-6 \chi_{g}}},
$$

where $\chi_{g}$ denotes the Euler characteristic, namely, $\chi_{g}=2-2 g$ in the orientable case and $\chi_{g}=2-g$ in the non-orientable case. For each case we denote by $R_{g}$ the radius satisfying equality in (1.1). A compact surface $S$ of genus $g$ is called an extremal surface if it admits an extremal disc, a disc of radius $R_{g}$. The hyperbolic surfaces are Riemann surfaces or non-orientable Klein surfaces. (For a reference of Klein surfaces, see, e.g., [1], 3], 13.) Let $\mathcal{K}_{g}$ be the moduli space of compact nonorientable Klein surfaces of genus $g$. We can then rephrase the radius $R_{g}$ of extremal discs in terms of injectivity radius $r_{p}(S)$ at a point $p$ of a compact non-orientable Klein surface $S$ as follows:

$$
R_{g}=\max _{S \in \mathcal{K}_{g}} \max _{p \in S} r_{p}(S) .
$$

In the case of Riemann surfaces the expression of $R_{g}$ is similar to (1.2) by using the moduli space $\mathcal{M}_{g}$ of compact Riemann surfaces of genus $g$.

Our problem here is to find how many extremal discs extremal surfaces can admit. For the orientable case, the problem is completely solved ([4, [5], [7], [],

Received by the editors October 10, 2015 and, in revised form, February 17, 2016.

2010 Mathematics Subject Classification. Primary 30F50; Secondary 30F40, 05C10.

This work was partially supported by the JSPS KAKENHI Grant No. 25400147. 
[12]). For the non-orientable case, we know that extremal surfaces of genus $g>6$ admit a unique extremal disc ([6]) and that those of genus 3,4 or 5 admit at most two $([6,[9$, [10], [11]). Those surfaces are presented by fundamental regions of their Fuchsian groups or non-Euclidean crystallographic groups (NEC groups). In the present paper we shall discuss non-orientable extremal surfaces of genus 6 , the final genus in our interest.

\section{MAin Results}

First we present our main results on non-orientable extremal surfaces of genus 6 .

Theorem 2.1. There exist 149, 279 non-orientable extremal surfaces of genus 6 up to isomorphism. They admit at most two extremal discs, and 107 of them admit exactly two extremal discs.

The first column of Table 1 shows the 107 surfaces. The surfaces $S_{j}$ are derived from the hyperbolic polygons $P_{j}$ in Figures 115, where lines and dotted lines in $P_{j}$ indicate pairs of sides identified by the opposite direction and the same direction, respectively. Note that we can take a regular $(6 g-6)$-gon as a fundamental region of an NEC group for a non-orientable extremal surface of genus $g$ and that the inscribed disc corresponds to an extremal disc ([2]). The second column of Table 1

TABLE 1. Extremal surfaces with two extremal discs. (Note that $S_{2} \cong S_{3}, S_{5} \cong S_{6}, S_{10} \cong S_{11}, S_{13} \cong S_{16}, S_{21} \cong S_{22}, S_{24} \cong S_{25}$, $S_{38} \cong S_{39}, S_{69} \cong S_{70}, S_{76} \cong S_{77}$.)

\begin{tabular}{lll}
\hline Surface & Centres of extremal discs & Aut $^{ \pm}$ \\
\hline$S_{2}, S_{5}, S_{10}, S_{13}, S_{21}, S_{38}, S_{69}, S_{76}$ & $\pi(0), \pi\left(\frac{2 i \sin 4 \beta}{\tanh R}\right)$ & $\{1\}$ \\
\hline$S_{17}$ & $\pi(0), \pi\left(\zeta_{17}\right)$ & $\mathbb{Z}_{2}$ \\
\hline$S_{28}, S_{29}, S_{34}, S_{35}, S_{80}, S_{81}, S_{82}$ & $\pi(0), \pi\left(\frac{i \sin 2 \beta}{\sin 3 \beta \tanh R}\right)$ & $\mathbb{Z}_{2} \times \mathbb{Z}_{2}$ \\
\hline$S_{30}, S_{31}, S_{49}, S_{50}, S_{83}, S_{85}, S_{88}$ & $\pi(0), \pi\left(\frac{1}{2 \sin 7 \beta \tanh R}\right)$ & $\mathbb{Z}_{2} \times \mathbb{Z}_{2}$ \\
\hline$S_{32}, S_{33}, S_{44}, S_{45}, S_{72}, S_{86}, S_{87}$ & $\pi(0), \pi\left(\frac{i}{2 \cos \beta \tanh R}\right)$ & $\mathbb{Z}_{2} \times \mathbb{Z}_{2}$ \\
\hline$S_{36}, S_{37}, S_{38}, S_{39}, S_{46}, S_{47}, S_{48}, S_{52}$, & $\pi(0), \pi\left(\frac{2 i \sin 4 \beta}{\tanh R}\right)$ & $\mathbb{Z}_{2} \times \mathbb{Z}_{2}$ \\
$S_{53}, S_{54}, S_{57}, S_{58}, S_{59}, S_{64}, S_{66}, S_{67}$, & $\pi(0), \pi\left(\frac{2 i \sin 4 \beta}{\tanh R}\right)$ & $D_{3}$ \\
$S_{69}, S_{70}, S_{78}, S_{79}, S_{84}, S_{89}, S_{90}, S_{94}$, & $\pi(0), \pi\left(\frac{2 i \sin 4 \beta}{\tanh R}\right)$ & $D_{3} \times \mathbb{Z}_{2}$ \\
\hline$S_{95}, S_{102}, S_{104}, S_{112}, S_{114}$ & $\mathbb{Z}_{2} \times \mathbb{Z}_{2}$ \\
\hline$S_{63}, S_{101}$ & $\pi(0), \pi\left(\frac{2 \sin 3 \beta}{\tanh R}\right)$ & $\mathbb{Z}_{2}$ \\
\hline$S_{68}, S_{103}$ & $\pi(0), \pi\left(\frac{2 i \sin 4 \beta}{\tanh R}\right)$ & \\
\hline$S_{91}, S_{92}, S_{96}, S_{97}, S_{98}, S_{111}, S_{115}$ & $\zeta_{17}=\frac{e^{18 \beta i} \sin 3 \beta+e^{11 \beta i} \tanh { }^{2} R \sin 2 \beta}{\sin 7 \beta \tanh R}$ \\
\hline The other 37 surfaces & $\pi(0)$
\end{tabular}



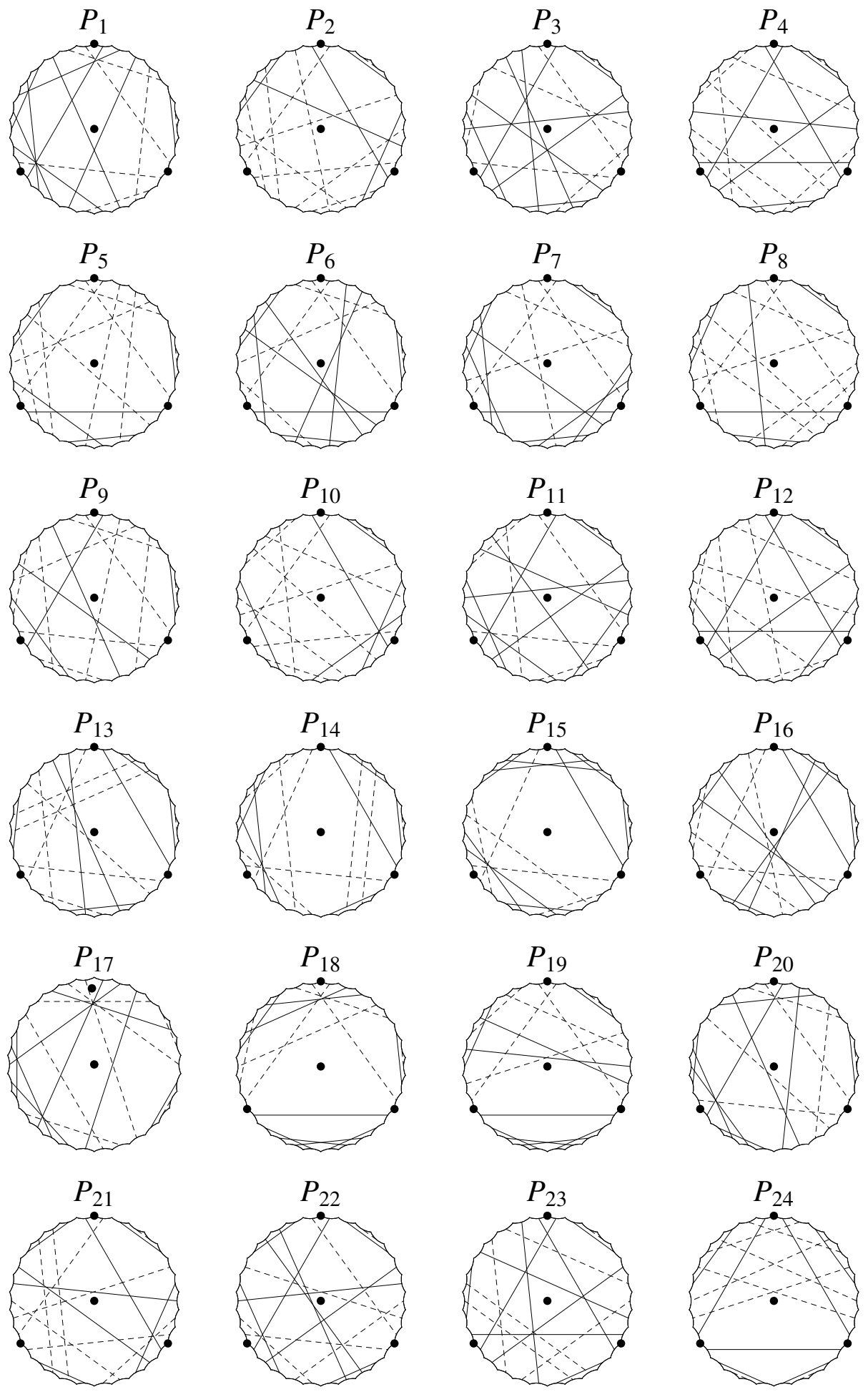

Figure 1. Side-pairing patterns and the centres of extremal discs $(\bullet)$ 

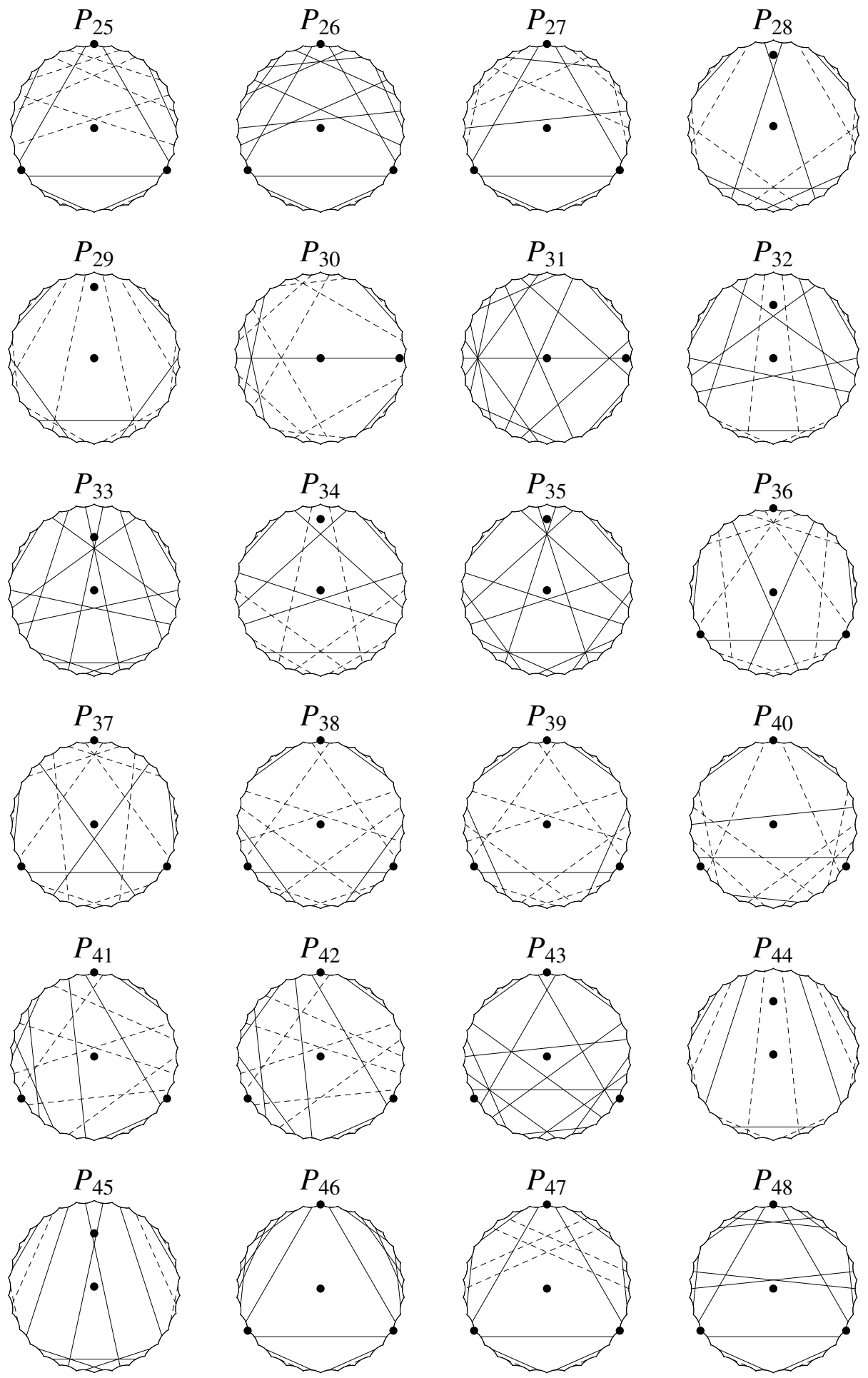

FiguRE 2. Side-pairing patterns and the centres of extremal discs $(\bullet)$ 

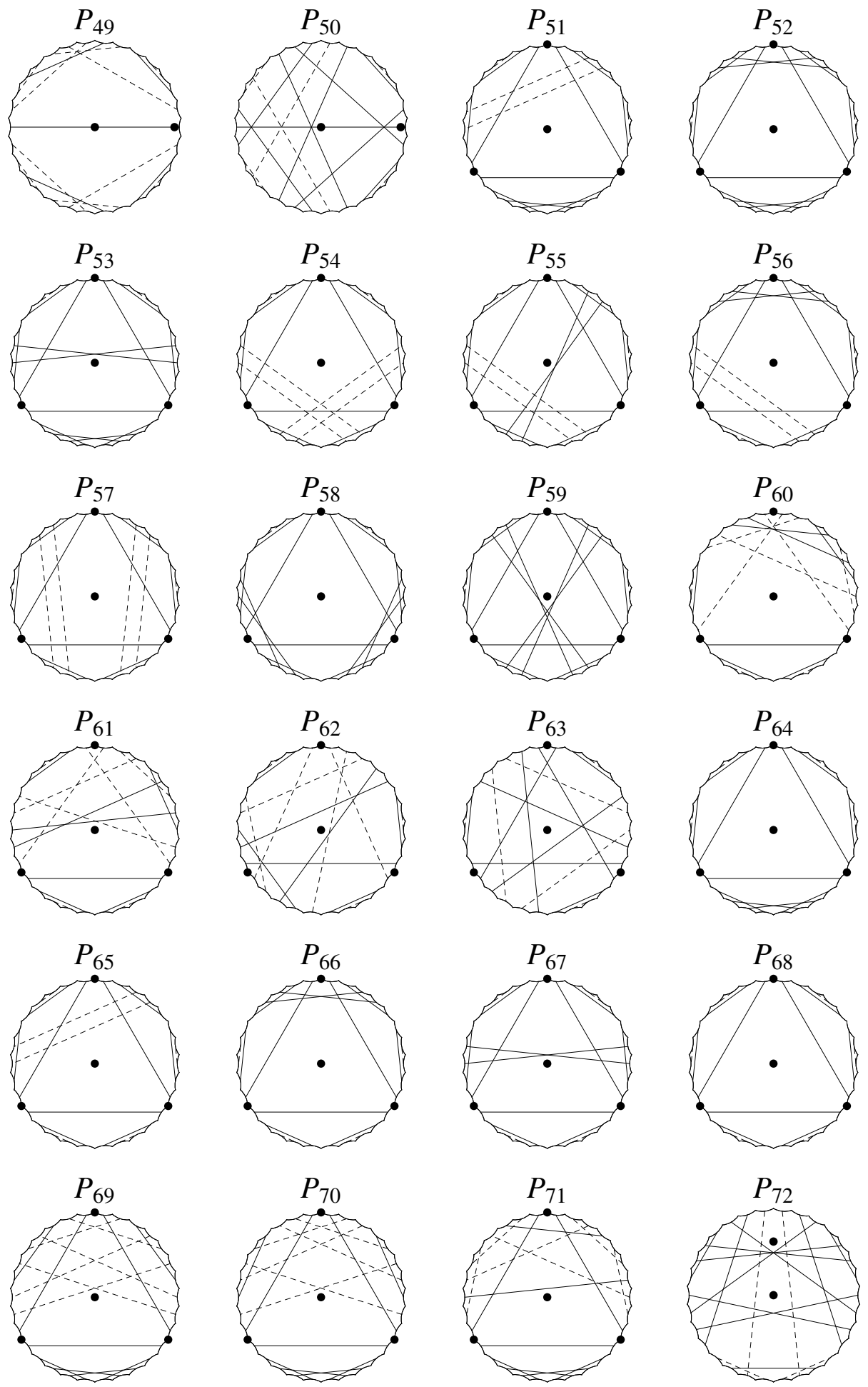

FiguRE 3. Side-pairing patterns and the centres of extremal discs $(\bullet)$ 

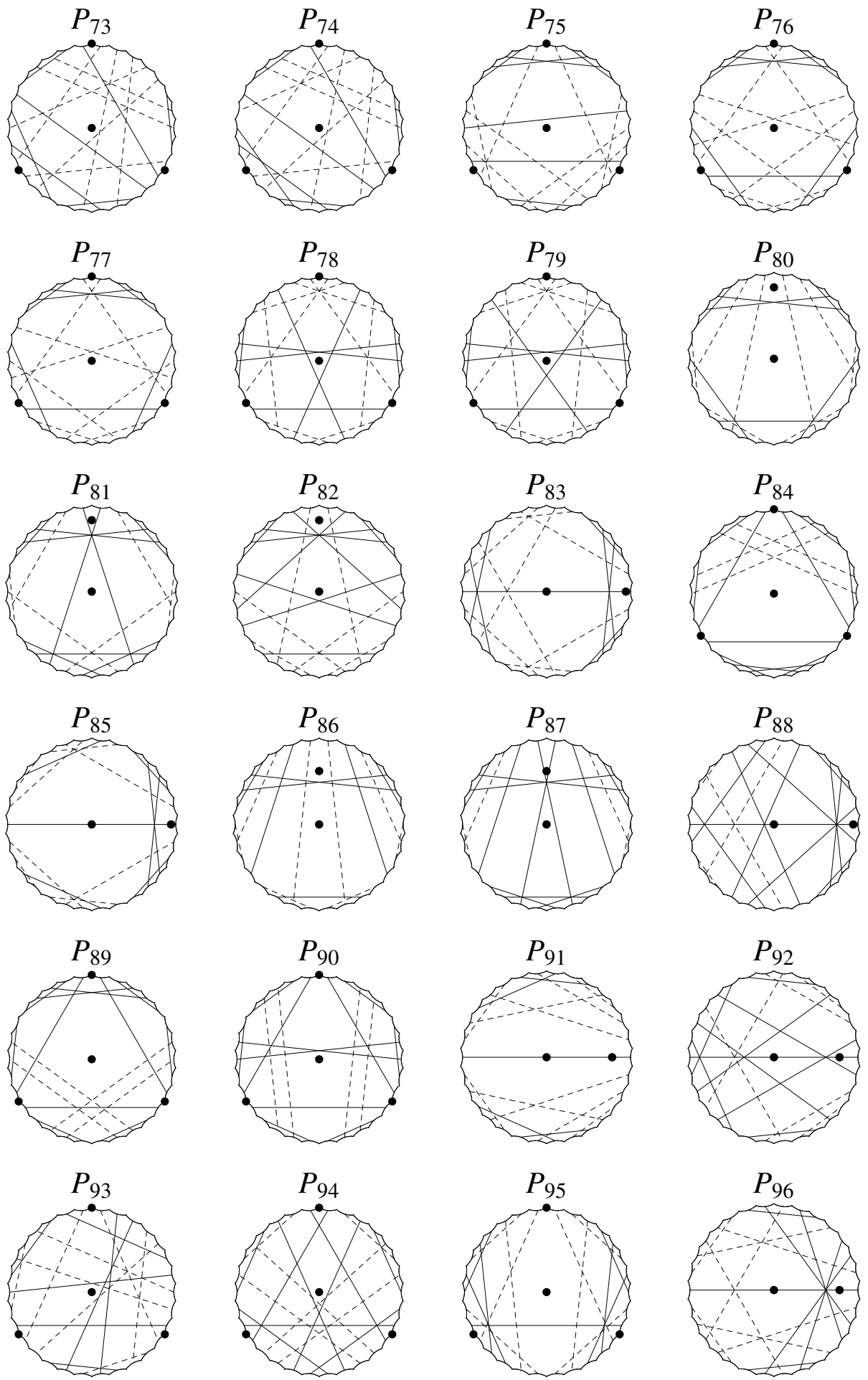

FiguRE 4. Side-pairing patterns and the centres of extremal discs $(\bullet)$ 

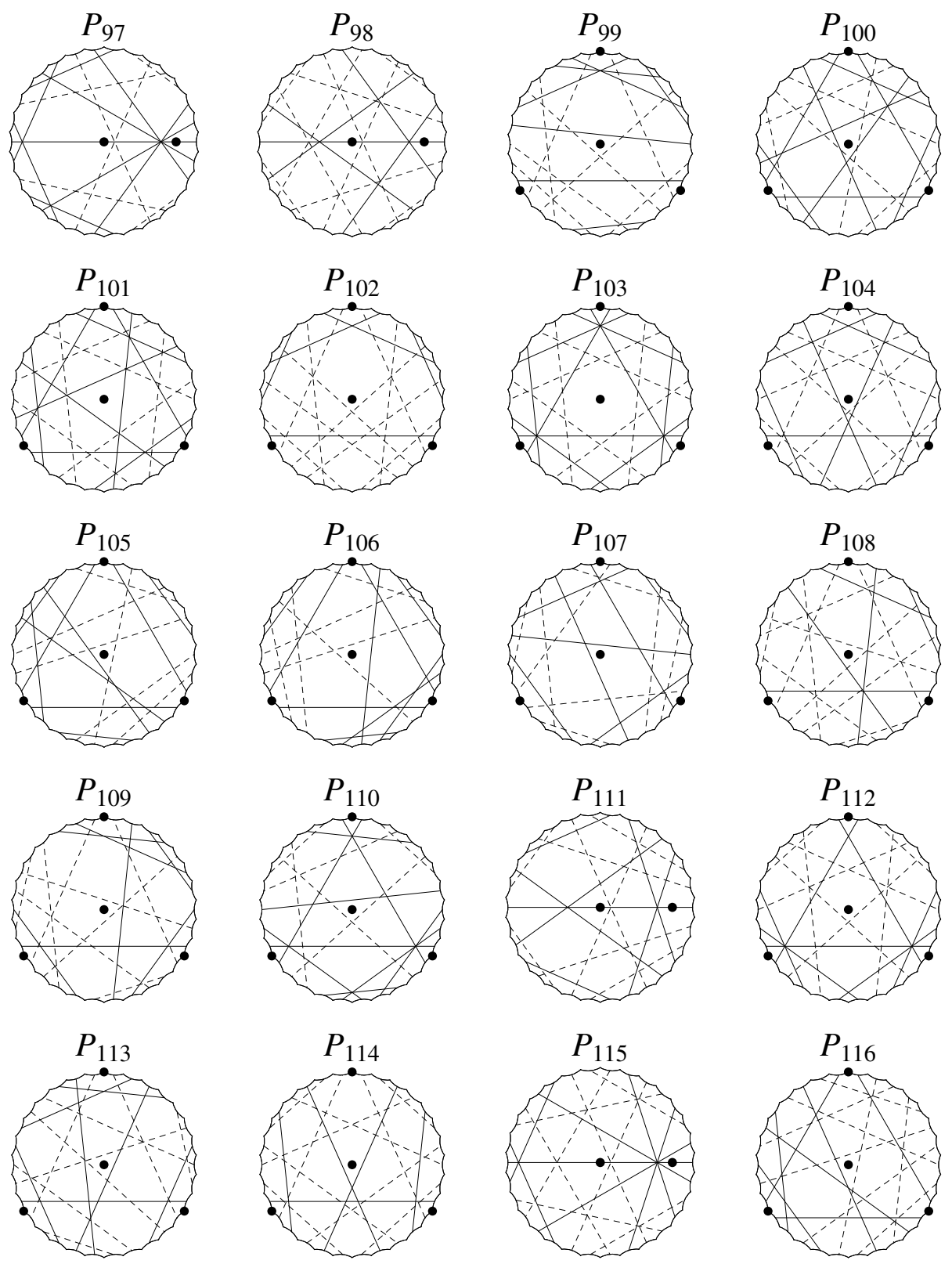

FiguRE 5. Side-pairing patterns and the centres of extremal discs

S $(\bullet)$

shows the loci of the centres of extremal discs, where $\pi\left(=\pi_{j}\right)$ denotes the natural projection from the unit disc $\mathbb{D}$ onto each surface $S_{j}, R=R_{6}$ and $\beta=\pi / 30$. To describe the centres we assume that the fundamental region is centrally located in $\mathbb{D}$ such that the vertices $v_{n}, n=1,2, \ldots, 30$, satisfy $\arg v_{n}=(2 n-1) \pi / 30$. The bullets in Figures 1,5 correspond to the centres of extremal discs. For example, $P_{2}$ has three bullets on the boundary. Since they project to the same point of $S_{2}$, 
a value $2 i \sin 4 \beta / \tanh R$ is selected as a representative and listed in Table 1. The third column of Table 1 shows the group of automorphisms $\operatorname{Aut}^{ \pm}\left(S_{j}\right)$ of $S_{j}$ in the category of Klein surfaces.

As a result of Theorem 2.1 and the results of $g \geqq 3(g \neq 6)([6,[9,[10,11)$ we have the following:

Theorem 2.2. Compact non-orientable hyperbolic surfaces of arbitrary genus admit at most 2 extremal discs.

\section{SidE-PAIRING PATTERNS OF 30-GON}

As mentioned in $₫ 1$, a non-orientable extremal surface of genus $g \geqq 3$ is represented by an NEC group of which a fundamental region is a regular $(6 g-6)$-gon in $\mathbb{D}$. We shall therefore consider a regular 30-gon for $g=6$. Since every angle of the polygon is equal to $2 \pi / 3$, three vertices project to one point on the surface. Therefore the boundary of the polygon projects to a trivalent graph with 10 vertices and 15 edges. A walk around the boundary of the polygon in one direction corresponds to a walk on the trivalent graph going through every edge twice. Conversely, for a given trivalent graph $G$, if we can walk continuously on every edge of $G$ twice, we obtain a side-pairing pattern of the polygon. Handling a computer, we see that there are 388 trivalent graphs with 10 vertices and 15 edges. Considering such a walk on each of those graphs, we see that there are 149,288 side-pairing patterns for the 30 -gon which becomes a non-orientable surface of genus 6 ; besides we obtain 927 side-pairing patterns which becomes an orientable surface of genus three.

Let $\mathcal{G}$ be the set of all trivalent graphs with 10 vertices and 15 edges. Since there are a lot of graphs, we shall classify $\mathcal{G}$ by introducing the terminology "loop" and "double" to a subgraph as depicted in Figure [6. Let $\mathrm{l} n \mathrm{~d} m$ be the subset of $\mathcal{G}$ with $n$ loops and $m$ doubles. For example, 11d4 consists of 4 graphs (Figure 7).

In Table 2 let TG denote the number of trivalent graphs; $\mathrm{SP}^{-}$the number of side-pairing patterns which make a non-orientable surface; and $\mathrm{SP}^{+}$the number of side-pairing patterns which make an orientable surface. In addition to that, we give the number of extremal surfaces derived from those side-pairing patterns: let $\mathrm{E}^{-}$denote the number of non-orientable extremal surfaces with more than one extremal disc; and $\mathrm{E}^{+}$the number of orientable extremal surfaces with more than one extremal disc.
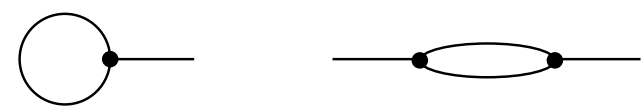

Figure 6. A loop and a double
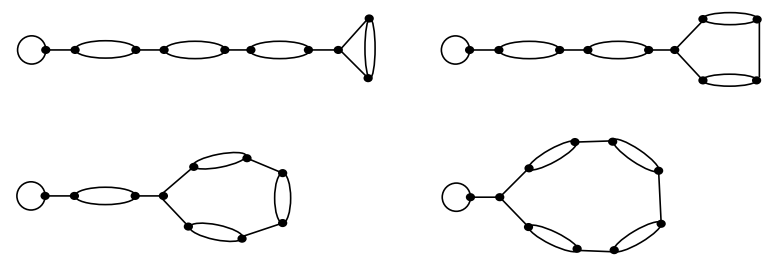

FiguRE 7. 11d4 (one loop and 4 doubles) 
TABLE 2. Trivalent graphs, induced side-pairing patterns, and induced extremal surfaces with more than one extremal disc.

\begin{tabular}{|r|rrrrr|}
\hline & TG & $\mathrm{SP}^{-}$ & $\mathrm{SP}^{+}$ & $\mathrm{E}^{-}$ & $\mathrm{E}^{+}$ \\
\hline $11 \mathrm{~d} 0$ & 19 & 20110 & 0 & 12 & 0 \\
$11 \mathrm{~d} 1$ & 33 & 18760 & 0 & 11 & 0 \\
$11 \mathrm{~d} 2$ & 32 & 7596 & 0 & 0 & 0 \\
$11 \mathrm{~d} 3$ & 18 & 1668 & 0 & 0 & 0 \\
$11 \mathrm{~d} 4$ & 4 & 114 & 0 & 0 & 0 \\
$12 \mathrm{~d} 0$ & 23 & 6050 & 0 & 20 & 0 \\
$12 \mathrm{~d} 1$ & 34 & 5178 & 0 & 0 & 0 \\
$12 \mathrm{~d} 2$ & 31 & 1912 & 0 & 16 & 0 \\
$12 \mathrm{~d} 3$ & 13 & 306 & 0 & 0 & 0 \\
$12 \mathrm{~d} 4$ & 1 & 6 & 0 & 0 & 0 \\
$13 \mathrm{~d} 0$ & 15 & 1086 & 0 & 4 & 0 \\
$13 \mathrm{~d} 1$ & 21 & 844 & 0 & 0 & 0 \\
$13 \mathrm{~d} 2$ & 16 & 270 & 0 & 0 & 0 \\
$13 \mathrm{~d} 3$ & 3 & 14 & 0 & 0 & 0 \\
$14 \mathrm{~d} 0$ & 9 & 162 & 0 & 0 & 0 \\
$14 \mathrm{~d} 1$ & 11 & 110 & 0 & 4 & 0 \\
$14 \mathrm{~d} 2$ & 5 & 19 & 0 & 0 & 0 \\
$15 \mathrm{~d} 0$ & 4 & 15 & 0 & 0 & 0 \\
$15 \mathrm{~d} 1$ & 3 & 7 & 0 & 0 & 0 \\
$16 \mathrm{~d} 0$ & 2 & 3 & 0 & 1 & 0 \\
$10 \mathrm{~d} 0$ & 19 & 33365 & 437 & 26 & 5 \\
$10 \mathrm{~d} 1$ & 23 & 32780 & 338 & 15 & 5 \\
$10 \mathrm{~d} 2$ & 25 & 14655 & 123 & 0 & 0 \\
$10 \mathrm{~d} 3$ & 16 & 3707 & 29 & 7 & 4 \\
$10 \mathrm{~d} 4$ & 7 & 535 & 0 & 0 & 0 \\
$10 \mathrm{~d} 5$ & 1 & 16 & 0 & 0 & 0 \\
& & & & & \\
Total & 388 & 149288 & 927 & 116 & 14 \\
\hline & & & & &
\end{tabular}

\section{LOCI OF EXTREMAL DISCS}

As mentioned in $\$ 1$, we shall normalize the hyperbolic regular 30-gon $P$ such that the centre is the origin and the vertices $v_{n}$ satisfy $\arg v_{n}=(2 n-1) \beta(n=$ $1, \ldots, 30)$, where $\beta=\pi / 30$. We denote by $C_{n}$ the side between $v_{n}$ and $v_{n+1}$ and by $w_{n}$ the middle point of $C_{n}$, where subscripts are regarded as modulo 30. By elementary calculation the two hyperbolic distances $l=d\left(0, v_{1}\right)$ and $s=d\left(v_{1}, v_{2}\right)$ are $l=\sinh ^{-1}\left(\frac{2}{\sqrt{3}} \sinh R\right) \approx 2.388$ and $s=2 \sinh ^{-1}\left(\frac{2}{\sqrt{3}} \sin \beta \sinh R\right) \approx 1.076$, where $R=R_{6}$ denotes the maximal radius satisfying (1.1) in $\$ 1$

Lemma 4.1. Let $S$ be a non-orientable extremal surface of genus 6 and $\pi: \mathbb{D} \rightarrow S$ the natural projection. If $p \in S$ is the centre of an extremal disc, then the hyperbolic distances between two points in the set $\pi^{-1}(p)$ are listed in increasing order such as $d_{1}=2 R \approx 4.494, d_{2} \approx 5.852, d_{3} \approx 6.642$, and so on. 
Proof. The elements of the list are calculated by considering the tessellation of $\mathbb{D}$ by hyperbolic regular 30 -gons. For example, $d_{2}=2 \sinh ^{-1}(\sinh 2 R \sin 2 \beta)$ and $d_{3}=2 \sinh ^{-1}(\sinh 2 R \sin 3 \beta)$.

Remark 4.2. The distance $d_{2}$ has another expression: $d_{2}=2 l+s([9$, Figure 2]).

Let $K_{n} \subset P(n=1, \ldots, 30)$ be the pentagon with vertices at $w_{n-1}, v_{n}, v_{n+1}$, $w_{n+1}$, and the origin.

Lemma 4.3. If a point $z$ in $K_{n}$ projects to the centre of an extremal disc, then it follows that $d\left(z, t_{n}(z)\right)=d_{1}$, where $t_{n}=t_{n, m}$ denotes an orientation preserving or reversing side-pairing mapping from $C_{n}$ onto the other side $C_{m}$.

Proof. Since $d\left(z, t_{n}(z)\right) \leqq \max \left\{d\left(v, t_{n}(v)\right) \mid v\right.$ is a vertex of $\left.K_{n}\right\}$, we shall estimate $d\left(v, t_{n}(v)\right)$. It is clear that $d\left(0, t_{n}(0)\right)=2 R ; d\left(v, t_{n}(v)\right) \leqq 2 l\left(v=v_{n-1}, v_{n}\right)$ because a vertex maps to the other vertex of $P$; and $d\left(v, t_{n}(v)\right) \leqq 2 R\left(v=w_{n-1}, w_{n}\right)$ because the midpoint of a side maps to the midpoint of the other side of $P$. Therefore $d\left(z, t_{n}(z)\right) \leqq 2 l<2 l+s=d_{2}$. Hence we have $d\left(z, t_{n}(z)\right)=d_{1}$.

If a point $z$ in $K_{n}$ projects to the centre of an extremal disc, then the equation $d\left(z, t_{n, m}(z)\right)=2 R$ implies that $z$ is on the curve $L_{n, m}$ or $M_{n, m}$ (resp. $L_{n, m}^{\prime}$ or $M_{n, m}^{\prime}$ ) if $t_{n, m}$ is orientation preserving (resp. reversing) (cf. [6, Lemma 5.5]):

$$
\begin{aligned}
& L_{n}=L_{n, m}:\left|z-\frac{\tanh R e^{i(n+m) \beta}}{2 \cos (n-m) \beta}\right|=\frac{\tanh R}{2|\cos (n-m) \beta|}(n-m \not \equiv 15(\bmod 30)), \\
& M_{n}=M_{n, m}: z=\operatorname{coth} R e^{2 i n \beta}-t e^{i(n+m+15) \beta}(t \in \mathbb{R}), \\
& L_{n}^{\prime}=L_{n, m}^{\prime}:\left|z-\frac{\operatorname{coth} R e^{i(n+m) \beta}}{2 \cos (n-m) \beta}\right|=\frac{\operatorname{coth} R}{2|\cos (n-m) \beta|}(n-m \neq 15(\bmod 30)), \\
& M_{n}^{\prime}=M_{n, m}^{\prime}: z=\tanh R e^{2 i n \beta}-t e^{i(n+m+15) \beta}(t \in \mathbb{R}) .
\end{aligned}
$$

We shall describe our process to find the centres of extremal discs for a surface $S_{j}$ :

(1) Take a polygon $P_{j}$. If a side-pairing mapping $t_{n}$ of $P_{j}$ is orientation preserving, then draw curves $L_{n}$ and $M_{n}$ on $K_{n}$, otherwise draw $L_{n}^{\prime}$ and $M_{n}^{\prime}$ on $K_{n}$ for every $n=1, \ldots, 30$.

(2) Find intersections of these curves on the subregion $K_{n} \cap K_{n+1}$ for every $n$.

(3) Choose every point $\zeta$ in the intersections such that the hyperbolic distance $d\left(\zeta, t_{k}(\zeta)\right)$ belongs to the list of Lemma 4.1 for every side-pairing mapping $t_{k}$ of $P_{j}$.

Applying this process to the 149,288 side-pairing patterns by computer, we see that only 116 of them, denoted by $P_{j}(j=1, \ldots, 116)$, yield two points (the origin and $\zeta \neq 0$ ) and that the others yield a unique point (the origin). For each of the patterns, except for 18 patterns $P_{2}, P_{3}, P_{5}, P_{6}, P_{10}, P_{11}, P_{13}, P_{16}, P_{21}, P_{22}, P_{24}$, $P_{25}, P_{38}, P_{39}, P_{69}, P_{70}, P_{76}, P_{77}$, we can show that the two points are transitive by a certain isometry $f$ of $\mathbb{D}$ which is compatible with the side-pairing mappings. Since we know that the origin projects to the centre of an extremal disc, so does the other point $\zeta$.

The following result holds for the 98 surfaces which are derived from the 116 patterns except for the 18 surfaces above.

Theorem 4.4. The 98 surfaces admit exactly two extremal discs. 
Though there are a lot of $P_{j}$ to be considered in this process, we shall give only two examples.

Example 4.5. We shall apply the process to $P_{1}$ (Figure 8). Then we get two

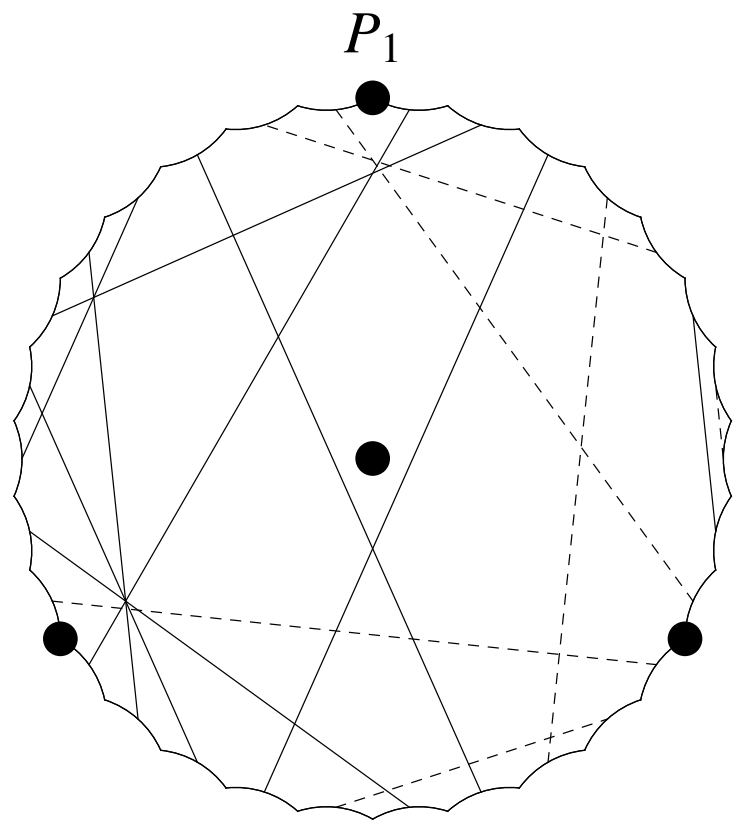

Figure 8. Side-pairing pattern $P_{1}$

points, the origin and $\zeta=2 i \sin 4 \beta / \tanh R$ (two more equivalent points $e^{2 \pi i / 3} \zeta$ and $e^{4 \pi i / 3} \zeta$ to be precise), where $\zeta$ is a solution of the simultaneous equations $M_{7,18}$ and $M_{8,28}^{\prime}$. Let $\alpha_{n, m}$ (resp. $\gamma_{n, m}$ ) denote the orientation preserving (resp. reversing) side-pairing mapping from $C_{n}$ onto $C_{m}$, that is,

$$
\alpha_{n, m}(z)=\frac{i \cosh R e^{i(m-n) \beta} z-i \sinh R e^{i(m+n) \beta}}{i \sinh R e^{-i(m+n) \beta} z-i \cosh R e^{-i(m-n) \beta}}
$$

and $\gamma_{n, m}(z)=\alpha_{-n, m}(\bar{z})$. Put $f(z):=(\zeta-z) /(1-\bar{\zeta} z)$; then we can verify that $f$ is compatible with the side-pairing mappings of $P_{1}$ as follows:

$$
\begin{array}{ll}
f \gamma_{1,30} f^{-1}=\gamma_{28,8} \gamma_{30,1} \gamma_{8,28}, & f \alpha_{2,29} f^{-1}=\gamma_{28,8} \gamma_{9,3}, \\
f \gamma_{3,9} f^{-1}=\gamma_{9,3}, & f \gamma_{4,25} f^{-1}=\gamma_{28,8} \alpha_{10,24}, \\
f \alpha_{5,21} f^{-1}=\alpha_{18,7} \alpha_{11,15}, & f \alpha_{6,13} f^{-1}=\alpha_{13,6}, \\
f \alpha_{7,18} f^{-1}=\alpha_{18,7}, & f \gamma_{8,28} f^{-1}=\gamma_{28,8}, \\
f \alpha_{10,24} f^{-1}=\gamma_{28,8} \gamma_{4,25}, & f \alpha_{11,15} f^{-1}=\alpha_{18,7} \alpha_{5,21}, \\
f \alpha_{12,19} f^{-1}=\alpha_{18,7} \alpha_{6,13}, & f \alpha_{14,20} f^{-1}=\alpha_{18,7} \alpha_{11,15} \alpha_{6,13}, \\
f \alpha_{16,23} f^{-1}=\gamma_{28,8} \gamma_{22,26} \alpha_{7,18}, & f \gamma_{17,27} f^{-1}=\gamma_{28,8} \alpha_{7,18}, \\
f \gamma_{22,26} f^{-1}=\gamma_{28,8} \alpha_{16,23} \alpha_{7,18} . &
\end{array}
$$

Consequently the projection $\pi(\zeta)$ of $\zeta$ is the centre of an extremal disc of the surface $S_{1}$, and $S_{1}$ admits exactly two extremal discs centred at $\pi(0)$ and $\pi(\zeta)$. 


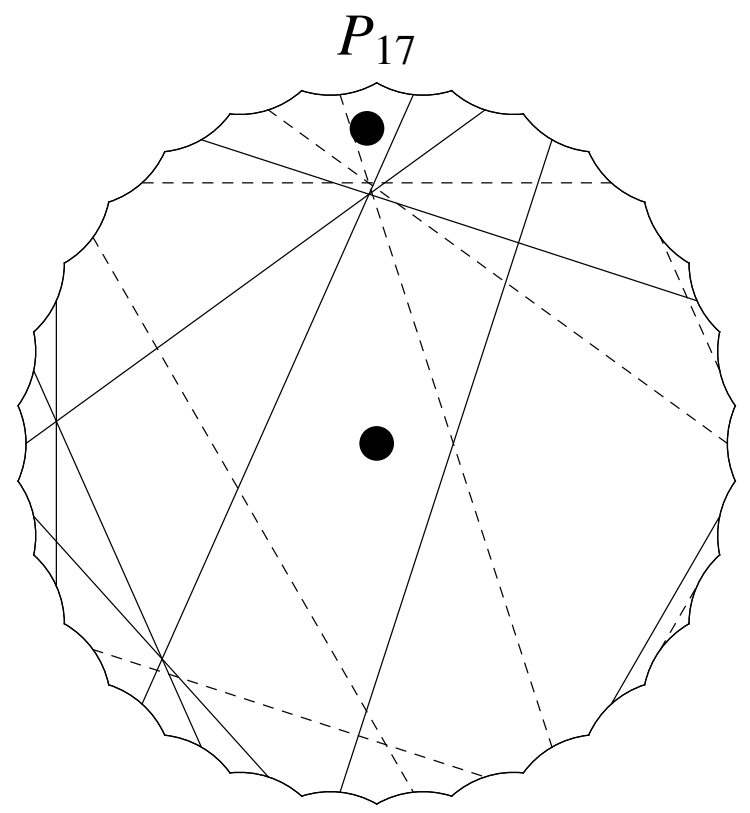

FiguRE 9 . Side-pairing pattern $P_{17}$

Example 4.6. We shall apply the process to $P_{17}$ (Figure 9). Then we get two points, the origin and $\zeta_{17}$, where

$$
\zeta_{17}=\frac{e^{18 \beta i} \sin 3 \beta+e^{11 \beta i} \tanh ^{2} R \sin 2 \beta}{\sin 7 \beta \tanh R}
$$

and $\zeta_{17}$ is a solution of the simultaneous equations $M_{7,19}$ and $M_{8,25}^{\prime}$. Put $f(z):=$ $\left(\zeta_{17}-z\right) /\left(1-\overline{\zeta_{17}} z\right)$; then we can verify that $f$ is compatible with the side-pairing mappings of $P_{17}$ as follows:

$$
\begin{array}{ll}
f \gamma_{1,3} f^{-1}=\alpha_{2,10} \gamma_{9,0}, & f \alpha_{2,10} f^{-1}=\alpha_{10,2}, \\
f \gamma_{4,11} f^{-1}=\gamma_{11,4}, & f \alpha_{5,22} f^{-1}=\gamma_{25,8} \gamma_{12,23}, \\
f \alpha_{6,15} f^{-1}=\alpha_{15,6}, & f \alpha_{7,19} f^{-1}=\alpha_{19,7}, \\
f \gamma_{8,25} f^{-1}=\gamma_{25,8}, & f \gamma_{9,30} f^{-1}=\gamma_{30,9}, \\
f \gamma_{12,23} f^{-1}=\gamma_{25,8} \alpha_{5,22}, & f \alpha_{13,17} f^{-1}=\alpha_{19,7} \alpha_{5,22}, \\
f \alpha_{14,20} f^{-1}=\alpha_{19,7} \alpha_{6,15}, & f \alpha_{16,21} f^{-1}=\alpha_{19,7} \alpha_{13,17} \alpha_{6,15}, \\
f \gamma_{18,24} f^{-1}=\gamma_{25,8} \alpha_{7,19}, & f \alpha_{26,29} f^{-1}=\gamma_{30,9} \gamma_{8,25}, \\
f \gamma_{27,28} f^{-1}=\gamma_{30,9} \gamma_{27,28} \gamma_{8,25} . &
\end{array}
$$

Consequently $\pi\left(\zeta_{17}\right)$ is the centre of an extremal disc of the surface $S_{17}$, and $S_{17}$ admits exactly two extremal discs centred at $\pi(0)$ and $\pi\left(\zeta_{17}\right)$.

For the exceptional cases, we have the following result.

Theorem 4.7. The surfaces $S_{2}$ and $S_{3}$ are isomorphic to each other. Also, $S_{5}$ and $S_{6} ; S_{10}$ and $S_{11} ; S_{13}$ and $S_{16} ; S_{21}$ and $S_{22} ; S_{24}$ and $S_{25} ; S_{38}$ and $S_{39} ; S_{69}$ and $S_{70}$; and $S_{76}$ and $S_{77}$ are isomorphic, respectively. Furthermore they admit exactly two extremal discs. 
Proof. We shall construct an isomorphism between $S_{2}$ and $S_{3}$. Let $f(z):=$ $\left(|\zeta|^{2}-\zeta \bar{z}\right) /\left(\bar{\zeta}-|\zeta|^{2} \bar{z}\right)$, where $\zeta=2 i \sin 4 \beta / \tanh R$ is a solution of the simultaneous equations $M_{7,17}^{\prime}$ and $M_{8,27}$. Then $f$ induces an isomorphism from $S_{2}$ onto $S_{3}$ because $f$ gives $S_{3}$ a regular 30-gon centred at $\zeta$ of which its side-pairing pattern is the same as that of $P_{2}$. In other words, the polygon $f\left(P_{2}\right)$ is a fundamental region of an NEC group of $S_{3}$ whose side-pairing mappings are generated by those of $P_{3}$. Therefore the projection $\pi(\zeta)$ is the centre of an extremal disc of $S_{2}$, and $S_{2}$ has exactly two extremal discs. We next show the relations between the side-pairing mappings of $P_{2}$ and those of $P_{3}$ (the right-hand sides are the compositions of sidepairing mappings of $P_{3}$ ):

$$
\begin{array}{ll}
f \alpha_{1,25} f^{-1}=\alpha_{18,7} \alpha_{1,15} \alpha_{6,3}, & f \gamma_{2,16} f^{-1}=\gamma_{28,8} \alpha_{6,3}, \\
f \alpha_{3,6} f^{-1}=\alpha_{6,3}, & f \gamma_{4,5} f^{-1}=\gamma_{5,4}, \\
f \gamma_{7,17} f^{-1}=\gamma_{28,8}, & f \alpha_{8,27} f^{-1}=\alpha_{18,7}, \\
f \gamma_{9,23} f^{-1}=\gamma_{28,8} \alpha_{13,26}, & f \alpha_{10,14} f^{-1}=\alpha_{22,9} \alpha_{10,24}, \\
f \gamma_{11,20} f^{-1}=\gamma_{28,8} \alpha_{10,24}, & f \alpha_{12,29} f^{-1}=\alpha_{18,7} \alpha_{9,22}, \\
f \gamma_{13,19} f^{-1}=\gamma_{28,8} \alpha_{9,22}, & f \gamma_{15,24} f^{-1}=\alpha_{18,7} \alpha_{1,15} \gamma_{8,28}, \\
f \gamma_{18,28} f^{-1}=\alpha_{18,7} \gamma_{8,28}, & f \gamma_{21,30} f^{-1}=\alpha_{18,7} \alpha_{25,21} \gamma_{8,28}, \\
f \gamma_{22,26} f^{-1}=\alpha_{18,7} \gamma_{16,12} \alpha_{7,18} . &
\end{array}
$$

The other cases are as follows:

$$
\begin{aligned}
& S_{5} \rightarrow S_{6}: f(z)=(\zeta-z) /(1-\bar{\zeta} z), \zeta=2 i \sin 4 \beta / \tanh R \text {. } \\
& f \gamma_{1,30} f^{-1}=\gamma_{28,8} \gamma_{30,1} \gamma_{8,28}, \quad f \alpha_{2,29} f^{-1}=\gamma_{28,8} \gamma_{9,3} \text {, } \\
& f \gamma_{3,9} f^{-1}=\gamma_{9,3} \text {, } \\
& f \gamma_{5,24} f^{-1}=\gamma_{28,8} \alpha_{11,25} \text {, } \\
& f \gamma_{7,17} f^{-1}=\gamma_{17,7} \\
& f \alpha_{10,14} f^{-1}=\alpha_{23,6} \alpha_{5,21} \text {, } \\
& f \gamma_{12,26} f^{-1}=\gamma_{28,8} \alpha_{6,23} \text {, } \\
& f \gamma_{4,15} f^{-1}=\gamma_{17,7} \alpha_{10,14}, \\
& f \gamma_{6,22} f^{-1}=\gamma_{28,8} \alpha_{13,26} \text {, } \\
& f \gamma_{8,28} f^{-1}=\gamma_{28,8} \text {, } \\
& f \gamma_{11,20} f^{-1}=\gamma_{17,7} \alpha_{5,21} \text {, } \\
& f \alpha_{16,23} f^{-1}=\gamma_{28,8} \alpha_{13,26} \gamma_{7,17}, \quad f \alpha_{18,27} f^{-1}=\gamma_{28,8} \gamma_{7,17} \text {, } \\
& f \alpha_{21,25} f^{-1}=\gamma_{28,8} \alpha_{20,24} \gamma_{7,17} \text {. } \\
& S_{10} \rightarrow S_{11}: f(z)=\left(|\zeta|^{2}-\zeta \bar{z}\right) /\left(\bar{\zeta}-|\zeta|^{2} \bar{z}\right), \zeta=2 i \sin 4 \beta / \tanh R \text {. } \\
& f \alpha_{1,25} f^{-1}=\alpha_{18,7} \alpha_{1,15} \alpha_{6,3}, \quad f \gamma_{2,16} f^{-1}=\gamma_{28,8} \alpha_{6,3}, \\
& f \alpha_{3,6} f^{-1}=\alpha_{6,3}, \\
& f \gamma_{7,17} f^{-1}=\gamma_{28,8} \\
& f \gamma_{9,13} f^{-1}=\gamma_{13,9}, \\
& f \gamma_{11,30} f^{-1}=\alpha_{18,7} \gamma_{10,21} \text {, } \\
& f \alpha_{14,20} f^{-1}=\gamma_{28,8} \alpha_{30,24} \gamma_{8,28} \text {, } \\
& f \gamma_{18,28} f^{-1}=\alpha_{18,7} \gamma_{8,28} \\
& f \gamma_{4,5} f^{-1}=\gamma_{5,4} \\
& f \alpha_{8,27} f^{-1}=\alpha_{18,7} \text {, } \\
& f \gamma_{10,21} f^{-1}=\gamma_{28,8} \alpha_{11,25} \text {, } \\
& f \gamma_{12,26} f^{-1}=\alpha_{18,7} \gamma_{9,13} \text {, } \\
& f \alpha_{22,29} f^{-1}=\alpha_{18,7} \alpha_{16,23} \alpha_{7,18} \text {. } \\
& f \gamma_{15,24} f^{-1}=\alpha_{18,7} \alpha_{1,15} \gamma_{8,28} \\
& f \gamma_{19,23} f^{-1}=\alpha_{18,7} \alpha_{23,16} \gamma_{8,28} \text {, } \\
& S_{13} \rightarrow S_{16}: f(z)=\left(|\zeta|^{2}-\zeta \bar{z}\right) /\left(\bar{\zeta}-|\zeta|^{2} \bar{z}\right), \zeta=2 i \sin 4 \beta / \tanh R \text {. } \\
& f \gamma_{1,30} f^{-1}=\alpha_{28,7} \gamma_{1,30} \alpha_{6,3}, \\
& f \alpha_{3,6} f^{-1}=\alpha_{6,3} \text {, } \\
& f \gamma_{5,14} f^{-1}=\gamma_{18,8} \alpha_{4,20}, \\
& f \gamma_{8,18} f^{-1}=\gamma_{18,8} \\
& f \alpha_{10,24} f^{-1}=\alpha_{28,7} \alpha_{11,25} \text {, } \\
& f \gamma_{12,26} f^{-1}=\alpha_{28,7} \gamma_{9,23} \text {, } \\
& f \gamma_{17,27} f^{-1}=\alpha_{28,7} \gamma_{8,18} \text {, } \\
& f \alpha_{2,29} f^{-1}=\alpha_{28,7} \alpha_{6,3}, \\
& f \gamma_{4,15} f^{-1}=\gamma_{18,8} \alpha_{5,21} \text {, } \\
& f \alpha_{7,28} f^{-1}=\alpha_{28,7} \text {, } \\
& f \alpha_{9,22} f^{-1}=\gamma_{18,8} \gamma_{12,16} \text {, } \\
& f \gamma_{11,20} f^{-1}=\gamma_{18,8} \alpha_{10,14} \text {, } \\
& f \alpha_{13,16} f^{-1}=\gamma_{18,8} \gamma_{9,23} \text {, } \\
& f \alpha_{21,25} f^{-1}=\alpha_{28,7} \gamma_{15,24} \gamma_{8,18} \text {. } \\
& f \gamma_{19,23} f^{-1}=\alpha_{28,7} \alpha_{13,26} \gamma_{8,18} \text {, }
\end{aligned}
$$


$S_{21} \rightarrow S_{22}: f(z)=\left(|\zeta|^{2}-\zeta \bar{z}\right) /\left(\bar{\zeta}-|\zeta|^{2} \bar{z}\right), \zeta=2 i \sin 4 \beta / \tanh R$.

$f \alpha_{1,25} f^{-1}=\alpha_{18,7} \alpha_{1,15} \alpha_{6,3}$

$f \gamma_{2,16} f^{-1}=\gamma_{28,8} \alpha_{6,3}$,

$f \alpha_{3,6} f^{-1}=\alpha_{6,3}$,

$f \gamma_{4,5} f^{-1}=\gamma_{5,4}$,

$f \gamma_{7,17} f^{-1}=\gamma_{28,8}$

$f \alpha_{8,27} f^{-1}=\alpha_{18,7}$,

$f \alpha_{9,12} f^{-1}=\alpha_{12,9}$,

$f \gamma_{10,21} f^{-1}=\gamma_{28,8} \alpha_{11,25}$,

$f \gamma_{11,20} f^{-1}=\gamma_{28,8} \alpha_{10,24}$,

$f \alpha_{13,26} f^{-1}=\alpha_{18,7} \alpha_{9,12}$,

$f \alpha_{14,30} f^{-1}=\alpha_{18,7} \gamma_{30,21} \gamma_{8,28}$,

$f \gamma_{15,24} f^{-1}=\alpha_{18,7} \alpha_{1,15} \gamma_{8,28}$,

$f \gamma_{18,28} f^{-1}=\alpha_{18,7} \gamma_{8,28}$,

$f \alpha_{19,22} f^{-1}=\gamma_{28,8} \alpha_{23,26} \gamma_{8,28}$,

$f \gamma_{23,29} f^{-1}=\alpha_{18,7} \gamma_{16,22} \alpha_{7,18}$.

$S_{24} \rightarrow S_{25}: f(z)=\left(|\zeta|^{2}-\zeta \bar{z}\right) /\left(\bar{\zeta}-|\zeta|^{2} \bar{z}\right), \zeta=2 i \sin 4 \beta / \tanh R$.

$f \alpha_{1,5} f^{-1}=\alpha_{30,4} \alpha_{7,28}$,

$f \gamma_{2,16} f^{-1}=\alpha_{17,8} \gamma_{6,12}$,

$f \gamma_{3,9} f^{-1}=\gamma_{6,12}$,

$f \gamma_{6,12} f^{-1}=\gamma_{3,9}$,

$f \gamma_{4,15} f^{-1}=\alpha_{17,8} \gamma_{5,14}$,

$f \alpha_{8,17} f^{-1}=\alpha_{17,8}$,

$f \gamma_{11,30} f^{-1}=\alpha_{28,7} \gamma_{10,1}$,

$f \alpha_{7,28} f^{-1}=\alpha_{28,7}$,

$f \alpha_{18,27} f^{-1}=\alpha_{28,7} \alpha_{8,17}$,

$f \alpha_{10,14} f^{-1}=\gamma_{3,9} \gamma_{10,1}$,

$f \gamma_{13,29} f^{-1}=\alpha_{28,7} \gamma_{9,3}$,

$f \gamma_{20,21} f^{-1}=\alpha_{17,8} \gamma_{21,20} \alpha_{8,17}$,

$f \alpha_{19,22} f^{-1}=\alpha_{17,8} \alpha_{22,19} \alpha_{8,17}$,

$f \gamma_{24,25} f^{-1}=\alpha_{28,7} \gamma_{25,24} \alpha_{7,28}$.

$S_{38} \rightarrow S_{39}: f(z)=\left(|\zeta|^{2}-\zeta \bar{z}\right) /\left(\bar{\zeta}-|\zeta|^{2} \bar{z}\right), \zeta=2 i \sin 4 \beta / \tanh R$.

$f \gamma_{1,20} f^{-1}=\gamma_{28,8} \alpha_{1,25} \alpha_{6,3}$,

$f \gamma_{2,16} f^{-1}=\gamma_{28,8} \alpha_{6,3}$,

$f \alpha_{3,6} f^{-1}=\alpha_{6,3}$,

$f \gamma_{4,5} f^{-1}=\gamma_{5,4}$,

$f \gamma_{7,17} f^{-1}=\gamma_{28,8}$

$f \gamma_{8,28} f^{-1}=\gamma_{17,7}$

$f \alpha_{9,12} f^{-1}=\alpha_{12,9}$,

$f \gamma_{13,29} f^{-1}=\gamma_{17,7} \alpha_{9,12}$,

$f \gamma_{10,11} f^{-1}=\gamma_{11,10}$,

$f \alpha_{15,21} f^{-1}=\gamma_{28,8} \alpha_{1,25} \gamma_{8,28}$,

$f \gamma_{14,25} f^{-1}=\gamma_{17,7} \gamma_{30,21} \gamma_{8,28}$

$f \gamma_{19,23} f^{-1}=\gamma_{17,7} \gamma_{23,19} \gamma_{8,28}$,

$f \alpha_{18,27} f^{-1}=\gamma_{17,7} \gamma_{8,28}$,

$f \alpha_{24,30} f^{-1}=\gamma_{17,7} \alpha_{20,14} \gamma_{7,17}$.

$f \gamma_{22,26} f^{-1}=\gamma_{17,7} \gamma_{19,23} \gamma_{7,17}$,

$S_{69} \rightarrow S_{70}: f(z)=\left(|\zeta|^{2}-\zeta \bar{z}\right) /\left(\bar{\zeta}-|\zeta|^{2} \bar{z}\right), \zeta=2 i \sin 4 \beta / \tanh R$.

$f \alpha_{1,5} f^{-1}=\alpha_{30,4} \alpha_{7,28}$,

$f \gamma_{2,16} f^{-1}=\alpha_{17,8} \gamma_{6,12}$,

$f \gamma_{3,9} f^{-1}=\gamma_{6,12}$,

$f \gamma_{6,12} f^{-1}=\gamma_{3,9}$,

$f \alpha_{8,17} f^{-1}=\alpha_{17,8}$,

$f \gamma_{11,30} f^{-1}=\alpha_{28,7} \gamma_{10,1}$,

$f \gamma_{4,15} f^{-1}=\alpha_{17,8} \gamma_{5,14}$,

$f \alpha_{7,28} f^{-1}=\alpha_{28,7}$,

$f \alpha_{18,27} f^{-1}=\alpha_{28,7} \alpha_{8,17}$,

$f \alpha_{10,14} f^{-1}=\gamma_{3,9} \gamma_{10,1}$,

$f \gamma_{13,29} f^{-1}=\alpha_{28,7} \gamma_{9,3}$,

$f \alpha_{20,24} f^{-1}=\alpha_{28,7} \alpha_{21,25} \alpha_{8,17}$,

$f \alpha_{19,22} f^{-1}=\alpha_{17,8} \alpha_{22,19} \alpha_{8,17}$,

$f \alpha_{23,26} f^{-1}=\alpha_{28,7} \alpha_{26,23} \alpha_{7,28}$.

$S_{76} \rightarrow S_{77}: f(z)=\left(|\zeta|^{2}-\zeta \bar{z}\right) /\left(\bar{\zeta}-|\zeta|^{2} \bar{z}\right), \zeta=2 i \sin 4 \beta / \tanh R$.
$f \gamma_{1,20} f^{-1}=\gamma_{28,8} \alpha_{1,25} \alpha_{6,3}$,
$f \gamma_{2,16} f^{-1}=\gamma_{28,8} \alpha_{6,3}$,
$f \alpha_{3,6} f^{-1}=\alpha_{6,3}$,
$f \alpha_{4,10} f^{-1}=\alpha_{5,11}$,
$f \alpha_{5,11} f^{-1}=\alpha_{4,10}$,
$f \gamma_{8,28} f^{-1}=\gamma_{17,7}$,
$f \gamma_{13,29} f^{-1}=\gamma_{17,7} \alpha_{9,12}$,
$f \alpha_{15,21} f^{-1}=\gamma_{28,8} \alpha_{1,25} \gamma_{8,28}$,
$f \gamma_{7,17} f^{-1}=\gamma_{28,8}$,
$f \alpha_{9,12} f^{-1}=\alpha_{12,9}$,
$f \gamma_{14,25} f^{-1}=\gamma_{17,7} \gamma_{30,21} \gamma_{8,28}$,
$f \gamma_{19,23} f^{-1}=\gamma_{17,7} \gamma_{23,19} \gamma_{8,28}$,
$f \alpha_{18,27} f^{-1}=\gamma_{17,7} \gamma_{8,28}$,
$f \alpha_{24,30} f^{-1}=\gamma_{17,7} \alpha_{20,14} \gamma_{7,17}$.
$f \gamma_{22,26} f^{-1}=\gamma_{17,7} \gamma_{19,23} \gamma_{7,17}$,

Hence we are done. 


\section{ISOMORPHICITY OF SURFACES}

Let $\mathcal{S}$ be the set of surfaces $S_{j}$ derived from all $P_{j}$ except for $j=3,6,11,16,22$, $25,39,70,77$. Therefore the cardinality of $\mathcal{S}$ is $\# \mathcal{S}=107$. We divide $\mathcal{S}$ into two parts $\mathcal{S}=\mathcal{S}_{1} \sqcup \mathcal{S}_{2}$, where $\mathcal{S}_{2}$ consists of the surfaces appearing in Theorem 4.7, i.e.,

$$
\mathcal{S}_{2}=\left\{S_{2}, S_{5}, S_{10}, S_{13}, S_{21}, S_{24}, S_{38}, S_{69}, S_{76}\right\}
$$

and $\mathcal{S}_{1}$ are the other surfaces in $\mathcal{S}$.

Theorem 5.1. For every surface in $\mathcal{S}_{1}$ two centres of extremal discs are transitive, that is, there exists an automorphism which maps one to the other. On the other hand, no surface in $\mathcal{S}_{2}$ has this property.

Proof. For the surfaces in $\mathcal{S}_{1}$, the statement is clear from our process to find that $\zeta(\neq 0)$ is the centre of an extremal disc. The isometry $f$ of $\mathbb{D}$ appearing in the process induces the required automorphism.

Let $S_{j}$ be a surface in $\mathcal{S}_{2}$ and let $S_{k}$ be the isomorphic surface with a side-pairing pattern $P_{k}$ different from $P_{j}$ (for example, $S_{3}$ for $S_{2}$ ). If we suppose that there exists an automorphism of $S_{j}$ which maps one centre to the other, then we can take an isomorphism $T: S_{j} \rightarrow S_{k}$ such that its lift $\tilde{T}: \mathbb{D} \rightarrow \mathbb{D}$ fixes the origin. Then $\tilde{T}$ must be a rotation around the origin or a reflection in a line passing through the origin. Such a mapping is incompatible with the side-pairing patterns $P_{j}$ and $P_{k}$. Therefore we have a contradiction.

Theorem 5.2. No surfaces in $\mathcal{S}$ are isomorphic to each other.

Proof. Case 1. Suppose that $S_{j} \in \mathcal{S}$ and $S_{k} \in \mathcal{S}_{1}$ are isomorphic $(j \neq k)$. Then there exists an isomorphism $T: S_{j} \rightarrow S_{k}$ satisfying $T\left(\pi_{j}(0)\right)=\pi_{k}(0)$ because the centres of extremal discs of $S_{k}$ are transitive. We can then take a lift $\tilde{T}: \mathbb{D} \rightarrow \mathbb{D}$ of $T$ which fixes the origin, so that we arrive at a contradiction in the same way as in the proof of Theorem 5.1 .

Case 2. Suppose that $S_{j} \in \mathcal{S}$ and $S_{k} \in \mathcal{S}_{2}$ are isomorphic $(j \neq k)$. Let $T$ : $S_{j} \rightarrow S_{k}$ be an isomorphism. If $T\left(\pi_{j}(0)\right)=\pi_{k}(0)$, then we have a lift $\tilde{T}: \mathbb{D} \rightarrow \mathbb{D}$ of $T$ which fixes the origin, a contradiction. If $T\left(\pi_{j}(0)\right)=\pi_{k}(\zeta)$, then we take a surface $S_{l}$ isomorphic to $S_{k}$ such that the side-pairing pattern $P_{l}$ is different from $P_{k}$. Let $F: S_{k} \rightarrow S_{l}$ be an isomorphism arising from the $f$ appearing in the proof of Theorem 4.7 which interchanges 0 and $\zeta$. Since $F\left(\pi_{k}(\zeta)\right)=\pi_{l}(0)$ hold, there exists an isomorphism $F \circ T: S_{j} \rightarrow S_{l}$ which maps $\pi_{j}(0)$ to $\pi_{l}(0)$. Hence we have a lift which fixes the origin, a contradiction again.

Corollary 5.3. There exist 149, 279 non-orientable extremal surfaces of genus 6 up to isomorphism.

Proof. There exist 149,288 side-pairing patterns. The 116 of them make the set $\mathcal{S}$ up to isomorphism, and $\# \mathcal{S}=107$. The other 149,172 patterns correspond to the non-orientable surfaces of genus 6 admitting a unique extremal disc. It is clear that they are not isomorphic to each other and not isomorphic to a surface in $\mathcal{S}$. Therefore we have 149,279 surfaces up to isomorphism.

\section{The groups of AUTOMORPHISMS OF SURFACES}

We shall consider the group of automorphisms for a surface in $\mathcal{S}$. In what follows, we use mappings $k: z \mapsto-\bar{z}$ and $r: z \mapsto e^{2 \pi i / 3} z$. 
$S_{2}$ : An automorphism $T$ of $S_{2}$ fixes the centres of extremal discs because $T$ does not interchange them (Theorem [5.1). We can take a lift $\tilde{T}: \mathbb{D} \rightarrow \mathbb{D}$ fixing the origin, so that it is a rotation around the origin or a reflection in a line passing through the origin. It is however incompatible with the side-pairing pattern of $P_{2}$ except for the identity mapping. Hence the group of automorphisms is trivial.

$S_{17}$ : There exists an automorphism $F$ induced by $f(z)=\left(\zeta_{17}-z\right) /\left(1-\overline{\zeta_{17}} z\right)$. Considering the side-pairing pattern of $P_{17}$ we see that an automorphism fixing $\pi(0)$ must be the identity mapping. Therefore $F$ is a unique automorphism interchanging the centres. Hence

$$
\operatorname{Aut}^{ \pm}\left(S_{17}\right)=\mathbb{Z}_{2}=\langle F\rangle
$$

$S_{28}$ : Let $f(z)=(\zeta-z) /(1-\bar{\zeta} z)$, where $\zeta=i \sin 2 \beta /(\sin 3 \beta \tanh R)$. Since $f$ and $k$ are compatible with the side-pairing pattern of $P_{28}$, they induce automorphisms $F$ and $K$ of $S_{28}$, respectively. All automorphisms are generated by $F$ and $K$ because each automorphism fixes or interchanges the centres of extremal discs. Since compositions of $f$ and $k$ satisfy $f^{2}=k^{2}=f k f k=i d$, we have

$$
\operatorname{Aut}^{ \pm}\left(S_{28}\right)=\mathbb{Z}_{2} \times \mathbb{Z}_{2}=\langle F\rangle \times\langle K\rangle .
$$

$S_{63}$ : Let $f(z)=\left(|\zeta|^{2}-\zeta \bar{z}\right) /\left(\bar{\zeta}-|\zeta|^{2} \bar{z}\right)$, where $\zeta=2 i \sin 4 \beta / \tanh R$. Then $f$ and $r$ induce automorphisms $F$ and $\sigma$ of $S_{63}$, respectively. All automorphisms are generated by $F$ and $\sigma$. Since $f^{2}=r^{3}=i d$ and $f r f r=\alpha_{27,8}$, we have

$$
\operatorname{Aut}^{ \pm}\left(S_{63}\right)=D_{3}=\left\langle F, \sigma \mid F^{2}=\sigma^{3}=1, F \sigma=\sigma^{-1} F\right\rangle .
$$

$S_{68}$ : Let $f(z)=(\zeta-z) /(1-\bar{\zeta} z)$, where $\zeta=2 i \sin 4 \beta / \tanh R$. Then $f, k$, and $r$ induce automorphisms $F, K$, and $\sigma$ of $S_{68}$, respectively. All automorphisms are generated by $F, K$, and $\sigma$. Since $k^{2}=r^{3}=k r k r=(f k)^{2}=(f k) k(f k) k=i d$ and $(f k) r(f k) r^{-1}=\alpha_{17,8}$, we have

$$
\operatorname{Aut}^{ \pm}\left(S_{68}\right)=D_{3} \times \mathbb{Z}_{2}=\langle K, \sigma\rangle \times\langle F K\rangle .
$$

$S_{101}$ : Let $f(z)=(\zeta-z) /(1-\bar{\zeta} z)$, where $\zeta=2 i \sin 4 \beta / \tanh R$. Then $f$ and $r$ induce automorphisms $F$ and $\sigma$ of $S_{101}$, respectively. All automorphisms are generated by $F$ and $\sigma$. Since $f^{2}=r^{3}=i d$ and $f r f r=\alpha_{28,7}$, we have

$$
\operatorname{Aut}^{ \pm}\left(S_{101}\right)=D_{3}=\langle F, \sigma\rangle \text {. }
$$

$S_{103}$ : Let $f(z)=(\zeta-z) /(1-\bar{\zeta} z)$, where $\zeta=2 i \sin 4 \beta / \tanh R$. Then $f, k$, and $r$ induce automorphisms $F, K$, and $\sigma$ of $S_{103}$, respectively. All automorphisms are generated by $F, K$, and $\sigma$. Since $k^{2}=r^{3}=k r k r=f^{2}=f k f k=i d$ and $f r f r^{-1}=\alpha_{18,7}$, we have

$$
\operatorname{Aut}^{ \pm}\left(S_{103}\right)=D_{3} \times \mathbb{Z}_{2}=\langle K, \sigma\rangle \times\langle F\rangle .
$$

The groups of automorphisms for the other surfaces are determined in a similar way.

From the arguments in $\S \$ 36$ we have Theorem 2.1 and Table 1

\section{ACKNOWLEDGMENTS}

The author gratefully acknowledges the helpful comments and suggestions of the referee in an earlier version of this paper. Finally, the author wishes to thank Institut de Mathématiques de Jussieu-Paris Rive Gauche, where the paper was written, for hospitality for a year. 


\section{REFERENCES}

[1] Norman L. Alling and Newcomb Greenleaf, Foundations of the theory of Klein surfaces, Lecture Notes in Mathematics, Vol. 219, Springer-Verlag, Berlin-New York, 1971. MR0333163

[2] Christophe Bavard, Disques extrémaux et surfaces modulaires (French, with English and French summaries), Ann. Fac. Sci. Toulouse Math. (6) 5 (1996), no. 2, 191-202. MR1413853

[3] Emilio Bujalance, José J. Etayo, José M. Gamboa, and Grzegorz Gromadzki, Automorphism groups of compact bordered Klein surfaces, Lecture Notes in Mathematics, vol. 1439, SpringerVerlag, Berlin, 1990. A combinatorial approach. MR.1075411

[4] Ernesto Girondo and Gabino González-Diez, On extremal discs inside compact hyperbolic surfaces (English, with English and French summaries), C. R. Acad. Sci. Paris Sér. I Math. 329 (1999), no. 1, 57-60, DOI 10.1016/S0764-4442(99)80461-3. MR1703263

[5] Ernesto Girondo and Gabino González-Diez, Genus two extremal surfaces: extremal discs, isometries and Weierstrass points, Israel J. Math. 132 (2002), 221-238, DOI 10.1007/BF02784513. MR.1952622

[6] Ernesto Girondo and Gou Nakamura, Compact non-orientable hyperbolic surfaces with an extremal metric disc, Conform. Geom. Dyn. 11 (2007), 29-43 (electronic), DOI 10.1090/S10884173-07-00157-9. MR2295996

[7] Gou Nakamura, The number of extremal disks embedded in compact Riemann surfaces of genus two, Sci. Math. Jpn. 56 (2002), no. 3, 481-492. MR.1937911

[8] Gou Nakamura, Extremal disks and extremal surfaces of genus three, Kodai Math. J. 28 (2005), no. 1, 111-130, DOI 10.2996/kmj/1111588041. MR2122195

[9] Gou Nakamura, Compact non-orientable surfaces of genus 4 with extremal metric discs, Conform. Geom. Dyn. 13 (2009), 124-135, DOI 10.1090/S1088-4173-09-00194-5. MR.2497316

[10] Gou Nakamura, Compact non-orientable surfaces of genus 5 with extremal metric discs, Glasg. Math. J. 54 (2012), no. 2, 273-281, DOI 10.1017/S0017089511000589. MR2911368

[11] Gou Nakamura, Compact Klein surfaces of genus 5 with a unique extremal disc, Conform. Geom. Dyn. 17 (2013), 39-46, DOI 10.1090/S1088-4173-2013-00251-8. MR3027523

[12] Gou Nakamura, Corrections to "Extremal disks and extremal surfaces of genus three" [MR2122195], Kodai Math. J. 37 (2014), no. 2, 475-480, DOI 10.2996/kmj/1404393899. MR.3229088

[13] Mika Seppälä and Tuomas Sorvali, Geometry of Riemann surfaces and Teichmüller spaces, North-Holland Mathematics Studies, vol. 169, North-Holland Publishing Co., Amsterdam, 1992. MR 1202043

Science Division, Center for General Education, Aichi Institute of Technology, YAKUSA-CHO TOYOTA 470-0392, JAPAN

E-mail address: gou@aitech.ac.jp 